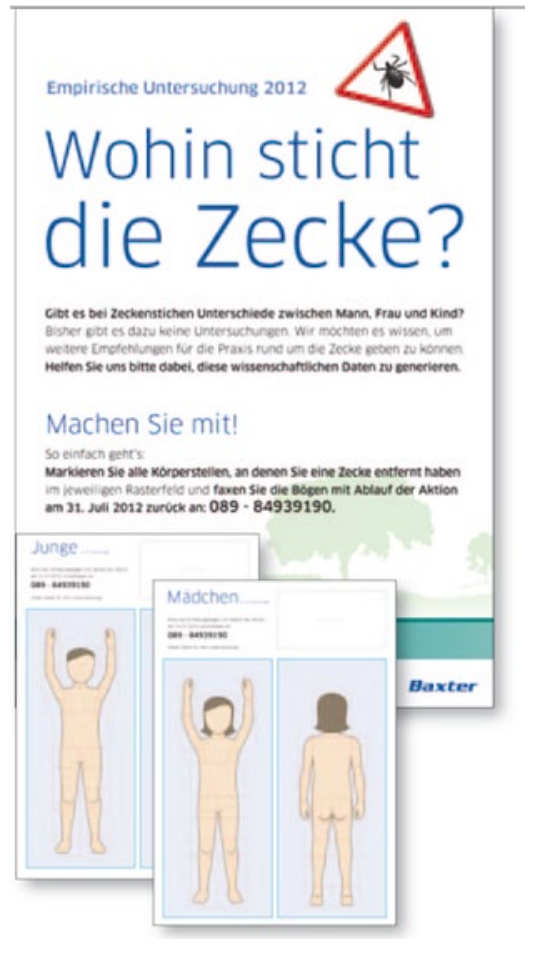

Machen Sie mit

\title{
Rasterfahndung nach der Zecke
}

- Bis heute gibt es keine systematischen Untersuchungen darüber, ob die in Deutschland mit Abstand häufigste Zeckenart Ixodes ricinus bestimmte Körperregionen für die anstehende tagelange Blutmahlzeit bevorzugt. Die bisherige Vermutung ist, dass bei Kindern vor allem die Kopfregion und bei Erwachsenen die Leistengegend betroffen sein können. Aber stimmt das? Gibt es Unterschiede zwischen den Geschlechtern wie bei der FSME-Manifestation, wo Männer doppelt so häufig an FSME erkranken wie Frauen? Oder sind Unterschiede zwischen Erwachsenen und Kindern an den Prädilektionsstellen für einen Zeckenstich signifikant feststellbar?

Diesen Fragen möchte der FSME- und Zeckenspezialist Baxter in Kooperation mit der Ärzte Zeitung in einer empirischen Untersuchung nachgehen.

\section{Ihre Unterstützung ist gefragt!}

Dazu benötigen die Wissenschaftler Ihre Unterstützung: Markieren Sie alle Körperstellen, an denen Sie eine Zecke entfernt haben auf einem Vordruck (s. Abb.). Diesen können Sie unter www.zecken.de (im Fachkreise-Bereich DocCheck-geschützt) als sechsseitiges PDF downloaden. Das Poster kann auch über Ihren Baxter-AußendienstMitarbeiter bezogen werden.

Faxen Sie die ausgefüllten Bögen bis zum 31. Juli 2012 an Baxter. Unter allen Teilnehmern werden 33 attraktive Preise verlost.

- Nach Informationen von Baxter

\section{Ungenügende Asthmakontrolle}

\section{Einer der Hauptgründe ist die mangelnde Therapieadhärenz}

- Das Ziel der Asthmatherapie, eine mehr oder weniger vollständige Kontrolle der Symptome, wird bei vielen Patienten trotz guter Therapiemöglichkeiten nicht erreicht. „Ärzte unterschätzen oft die Symptomatik von Asthmapatienten und damit den Grad der Asthmakontrolle", berichtete Dr. Christian Virchow, Rostock. Viele Patienten werden nach KV- und Krankenkassendaten in Deutschland zudem nicht leitliniengerecht behandelt. Insbesondere die Therapiepersistenz bei der Einnahme von Controllersubstanzen wie inhalierbaren Kortikosteroiden (ICS) und lang wirksamen Beta-2-Agonisten (LABAs) lässt zu wünschen übrig, wie Zahlen der KV Bayern zu Verordnungen zwischen dem 1. April 2005 und dem 31. März 2006 bei mehr als 483000 Asthmapatienten belegen. Nur rund $60 \%$ der Patienten - zwei Drittel wurden von Hausärzten behandelt - erhielten Verschreibungen von Asthmamedikamenten, berichtete Virchow, und Controllersubstanzen wurden im Beobachtungszeitraum von einem Jahr bei Berücksichtigung der definierten täglichen Dosis (DDDs) im Mittel nicht länger als 90 Tage eingesetzt (Hasford J et al. Allergy 2010; 65: 347-354).

\section{Inhalative Therapie oft fehlerhaft}

Eine ungenügende Asthmakontrolle kann auch an der Non-Compliance der Patienten liegen: fehlende Einlösung von Rezepten, bedarfsweise statt regelmäßige Inhalation von Controllermedikamenten oder fehlerhafte inhalative Therapie. Im Mittel benutzt fast jeder dritte erwachsene Patient Trockenpulverinhalatoren (DPI) fehlerhaft, berichtete Dr. Thomas Voshaar, Moers. Für viele Patienten sind treibgasgetriebene Dosieraerosole geeigneter als DPI, weil die Lungendeposition weniger abhängig von Inspirationsfluss und -volumen ist. Besonders günstig für eine stärkere Partikeldeposition in der Peripherie des Bronchialbaums sind extrakleine Partikel mit einem Durchmesser von 1-2 $\mu$ m, wie sie vom HFA-Dosieraerosol Inuvair ${ }^{\circledR}$ Mikrosol mit Formoterol und Beclometason in Lösung freigesetzt werden.
- Roland Fath

Quelle: Symposium „Therapieziel Asthmakontrolle - Strategien für eine optimierte Versorgung", Kongress der Deutschen Gesellschaft für Pneumologie und Beatmungsmedizin, Nürnberg , März 2012 (Veranstalter: JanssenCilag)

\section{Kurz notiert}

Neue Packungsgrößen - Frag$\min ^{\circledast}$ Fertigspritzen werden mit sieben neuen Packungsgrößen und in neuem Design angeboten: zur Prophylaxe tiefer Venenthrombosen: Fragmin $^{\oplus} \mathrm{P}$ (2.500 I.E.) und Fragmin ${ }^{\circledast}$ P Forte (5.000 I.E.) mit jeweils 50 Fertigspritzen; zur Therapie akuter tiefer Venenthrombosen und Lungenembolien, zur Rezidivprophylaxe venöser Thromboembolien bei onkologischen Patienten: Fragmin ${ }^{\circledR} 7.500$ I.E./10.000 I.E./12.500 I.E./15.000 I.E. und 18.000 I.E. mit jeweils 20 Fertigspritzen.

Pfizer 